\title{
Trajectories of cotton fiber initiation: a regulatory perspective
}

Pravin Prakash', Rakesh Srivastava', Priti Prasad ${ }^{1,2}$, Vipin Kumar Tiwari ${ }^{1,2}$, Ajay Kumar $^{1}$, Shatrujeet Pandey ${ }^{1}$ and Samir V. Sawant ${ }^{1,2 \bowtie}$

${ }^{1}$ Molecular Biology and Biotechnology Division, Council of Scientific and Industrial ResearchNational Botanical Research Institute (CSIR-NBRI), Lucknow - 226001, Uttar Pradesh, India

${ }^{2}$ Academy of Scientific and Innovative Research (AcSIR), Ghaziabad - 201002, Uttar Pradesh, India.

${ }^{\circledR}$ Corresponding author

\section{Dr. Samir V. Sawant}

Senior Principal Scientist

Molecular Biology and Biotechnology Division

CSIR-National Botanical Research Institute

Rana Pratap Marg, Lucknow - 226001, Uttar Pradesh, India

E-mail: samirsawant@nbri.res.in 


\section{Highlights}

- Cotton fiber is a major cash crop worldwide.

- The cotton fiber initiation is a complex biological process. Several genes, transcription factors, epigenetic regulators, small RNAs, and phytohormones coordinate the regulatory events, which lead to fiber initiation.

- The fiber initiation phase plays a crucial role in determining the commitment of ovule epidermal cells to fiber-producing or non-fiber producing cells.

- The precise understanding of the molecular events that govern fiber initiation is essential to genetically modulate the fiber quantity and quality. 


\begin{abstract}
The epidermal cells on the surface of the cotton ovules undergo differentiation to produce fibers, which are single-celled hair-like protrusions resembling the plant trichomes. The initiation of these unicellular fibers from the cotton ovule surface is a complex and tightly regulated process. The initiation step is the cell fate-determining stage, which leads to the commitment of cells that eventually developed into fibers, thus becomes the most crucial phase in fiber development. The in-depth knowledge of molecular regulation is a prerequisite to get a clear view of the fiber initiation process's genetic and epigenetic control. The identification and functional validation of cotton fiber initiation-related genes, few fibreless mutants, transcription factors, microRNAs, epigenetic regulators, as well as the elucidation of the role of phytohormones as signaling molecules, has played a significant role in understanding the cotton fiber initiation process at the molecular level. This review focuses on the comprehensive information regarding the genetic and epigenetic regulation of cotton fiber initiation. Thus, the review will provide readers insight into mechanistic details that operate during cotton fiber initiation.
\end{abstract}

Key words: Cotton, Fiber initiation, Genomics, Epigenomics, Phytohormones, Transcription factors, MicroRNAs, Gene expression regulation 


\section{Introduction}

The domestic and industrial demand for natural fiber ranks the cotton first among the cash crops worldwide. Considering the global demand for cotton fibers, the most crucial aspect of cotton research is the enhancement of the fiber yield and quality. The technological advancements in the post-genomic era have greatly facilitated to unravel the complex molecular mechanisms involved in cotton fiber development. The cotton fiber producing cells begin to differentiate over the ovule epidermal surface around 1-3 days before anthesis. Subsequent progress in these initial events leads to the emergence of fiber initials over the ovule epidermis, usually at anthesis (Stewart 1975; Graves and Stewart, 1988). The post-initiation stages during the fiber cell differentiation and development include the elongation, secondary cell wall biosynthesis, and maturation phases. The fiber initials start to protrude from the chalazal end of the ovule before the anthesis, whereas at the micropylar end, very few or no fiber initials emerge until the anthesis (Berkley 1948; Basra and Malik, 1984). It is the initiation phase during which the cotton ovule epidermal cells are committed to develop into the fiber cells or the non-fiber cells. The committed ovule epidermal cells differentiate and develop into two morphologically distinct fiber cells: lint, and linters or fuzz (Figure 1). Around $25 \%$ of the committed ovule epidermal cells differentiate into the lint fibers. Compared to the short linters $(<15 \mathrm{~mm}$ in length) or the fuzz fibers, the elongated lint fibers ( $\sim 30 \mathrm{~mm}$ in length) on the cottonseed coat are economically significant, since these long lint fibers are spinnable into yarn (Basra and Malik, 1984; Kim and Triplett, 2001). There are time-dependent developmental waves that determine the emergence and development of lint or fuzz fibers from the ovule epidermal cells. The ovule epidermal cells are committed on or before the day of anthesis to produce the fibers of commercial interest i.e., the lint fibers. Although the fuzz fibers appear on the ovule surface after a few days, but it 


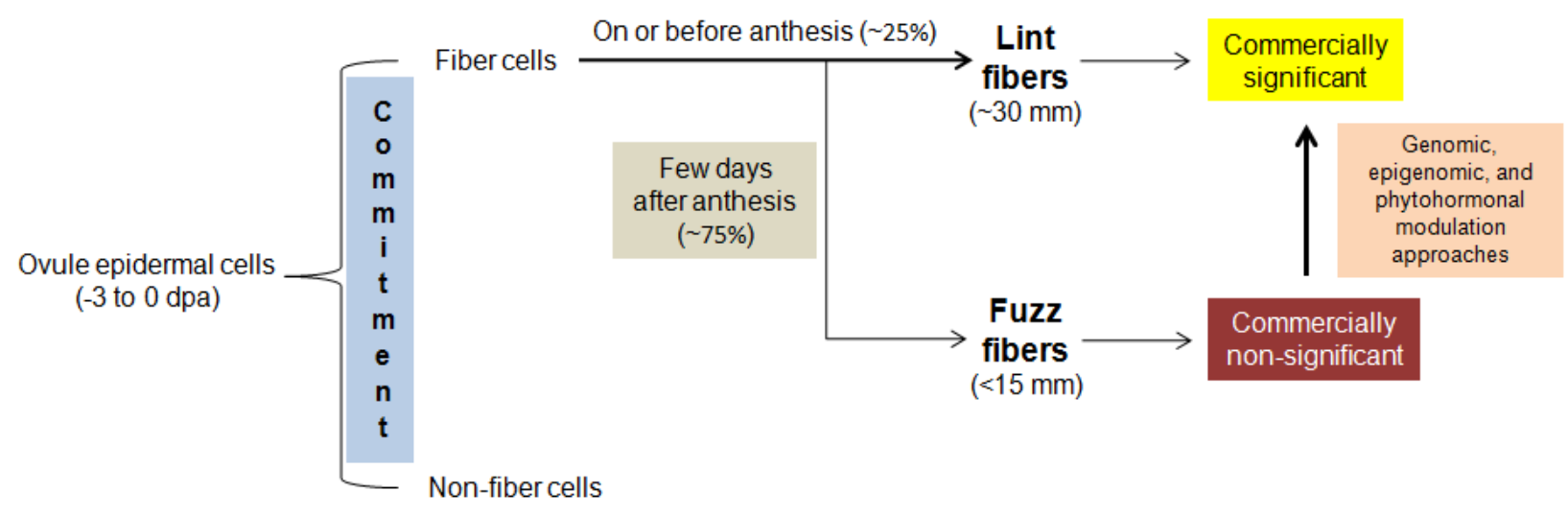

Figure 1. The major events during cotton fiber initiation leading to the production of lint or fuzz fibers. The genomic, epigenomic, and phytohormonal modulation approaches hold promise to enhance the production of commercially important lint fibers.

appears that their fate is determined during the initiation phase. The initiation phase appears to be tightly regulated at the molecular level to decide the fate of the nascent ovule epidermal cells. There appear to be genes, transcription factors (TFs), epigenetic factors, small RNAs (sRNAs), and the phytohormones governing the precise regulation of the cotton fiber initiation (Figure 2). Although many cotton fiber initiation-related genetic and epigenetic factors and the phytohormones have been identified and characterized, many questions are still unanswered concerning the molecular regulation of the commitment of cotton ovule epidermal cells during the fiber initiation stage. Thus, the review's main focus is to provide a comprehensive account of the potential genetic and epigenetic factors, and the phytohormones which modulate the molecular events and possibly be involved in determining the commitment of the ovule epidermal cells to develop into the fiber cells. 


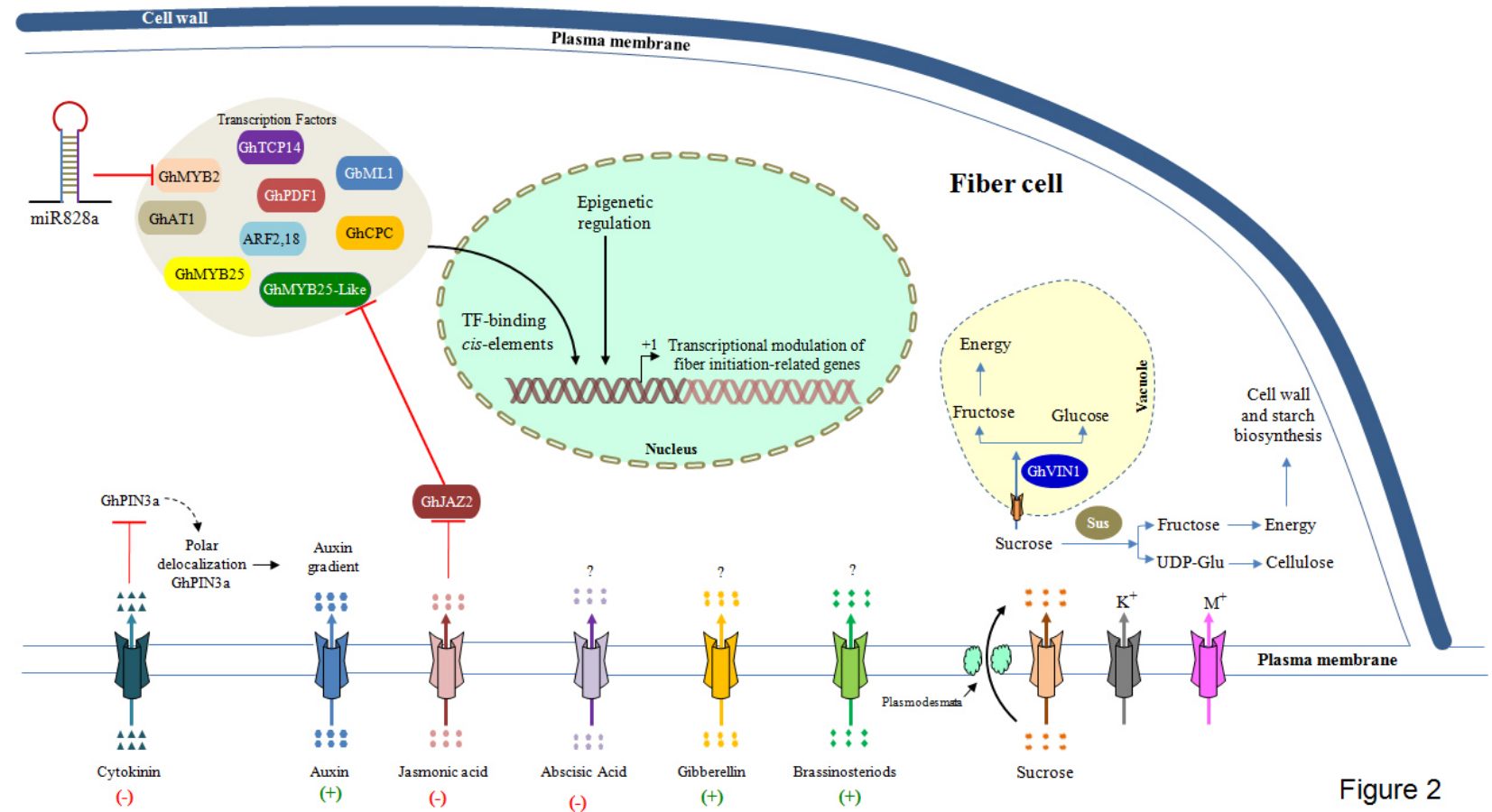

Figure 2. Schematic model showing the interplay of potential genetic, epigenetic regulators, and phytohormones facilitating the initiation of cotton fibers over the ovule epidermal surface. The green colored (+) signs and red colored (-) signs represent the phytohormone-mediated positive and negative regulation, respectively.

\section{The genes display a potential involvement in cotton fiber initiation}

The identification and experimental validation of genes that are preferentially expressed during the fiber initiation phase has significantly contributed to understand the molecular aspects of cotton fiber initiation. Since the beginning of fiber initiation research, several molecular approaches have assisted in the identification of genes involved in the regulation of cotton fiber initiation. However, the current technological advances including the high-throughput sequencing technologies have greatly facilitated to unravel the genes that play important roles during the fiber initiation processes. The isolation, functional annotation, and expression profiling of genes during the early stages of fiber development could infer their potential involvement in the fiber initiation-specific processes. In an earlier study, the gene expression 
analyses during the fiber initiation stages in cotton genotype Texas Marker-1 (wild type), and naked seed mutant (N1N1) lines revealed the probable involvement of genes such as protodermal factor 1 (PDF1), MYB25, and dehydration-induced protein RD22-like protein 1 (RDL1) in cotton fiber initiation (Lee et al., 2006). In an another study, the gene expression analyses by using cDNA microarrays and laser capture microdissection (LCM) of fiber cells in 0 days post-anthesis (DPA) ovules from wild type cotton and fiberless mutants, revealed several potential genes differentially expressed during the lint fiber initiation (Wu et al., 2006). These include GhMyb25, GhMyb25-like, homeodomain protein1 (GhHD1), cyclinD3;1(GhCycD3;1), sucrose synthase (GhSus), $\alpha$-expansin, and lipid transfer protein (GhLTP). Among these genes, GhMyb25, GhMyb25-like, and GhHD1 are transcriptional regulators, whereas, the GhCycD3;1 is involved in the cell cycle regulation. Most of the fiber cells might undergo DNA endoreduplication during their development (Wu et al., 2006). Being the highly expressed cell cycle regulatory gene, the $G h C y c D 3 ; 1$, might be an important candidate gene involved in DNA endoreduplication during the cotton fiber initiation (Wu et al., 2006). Similarly, the cDNA expression analysis of -2 to +25 DPA ovule and fiber tissues of Gossypium barbadense cv. Pima 3-79 showed the highest levels of G. barbadense gibberellin 20-oxidase 1 (GbGA20oxl) transcripts at 0 DPA (Tu et al., 2007). The expression pattern of GbGA20ox1 resembled with $G$. hirsutum gibberellins 20-oxidase 1 (GhGA20oxl) gene, as reported previously by Shi et al. (2006). It plays an important role in gibberellin (GA)-mediated fiber initiation in G. barbadense (Tu et al., 2007). The gene expression analyses showed the undetectable levels of sucrose synthase encoding gene $(S u S y)$ transcripts in fiberless seed $(f l s)$ mutants on the day of anthesis compared to the wild type. Concurrent to the expression of $S u S y$, the fls mutant ovules also lack the fiber initials on the day of anthesis, compared to the wild type (Ruan and Chourey, 1998). 
The suppression of the sucrose synthase (Sus) gene activity also resulted in the fiber growth inhibition over the ovule epidermal cells (Ruan et al., 2003). The MYB2, MYB109, and genes similar to Arabidopsis thaliana GLABRA2 (GL2) and CAPRICE/TRIPTYCHON (CPC), preferentially expressed in +1 DPA fiber initials and speculated to play an important role in cotton fiber initiation (Taliercio and Boykin, 2007). The gene expression analyses in JKC777 (superior fiber producing variety), and JKC703 (inferior fiber producing variety) led to the identification of differentially expressed genes at the 0 DPA stage in the fiber cells (Nigam et al., 2014). Genes that showed preferential expression during the initiation phase in the superior variety include sucrose synthase 4 (GhSUS4), cellulose synthase-like A3 (GhCSLA03), 3ketoacyl-CoA synthase 6 (GhKCS6), flavanone 3' hydroxylase (GhF3'H), GhMYB25, GhMYB109, MYB3, MYB109, MYB26, MYB73, and MYB4 (Nigam et al., 2014). The role of $G$. hirsutum vacuolar invertase (VIN) gene, GhVIN1, was investigated in fiber initiation by Wang et al. (2014a) through the RNA interference (RNAi)-mediated suppression line, which ultimately showed the fiberless seed phenotype in transgenic cotton. The GhVINI-mediated hexose signaling plays an important role in fiber initiation by acting upstream to the pathway, which involves the GhMYB25-like, GhMYB25, and GhMYB109 TFs, and the auxin response genes, viz. GhABP1, GhARF1, GhARF3, and GhbHLH (Wang et al., 2014a). G. hirsutum 3-hydroxyacylCoA dehydratase PASTICCINO 2(GhPAS2) gene function was investigated in cotton fiber initiation by Wang et al. (2015a). GhPAS2 play a vital role in very-long-chain fatty acid (VLCFA) biosynthesis and its expression was maximum at the day of anthesis in the TM1 (wild type) ovules. The fiber cell protrusion was suppressed upon the application of VLCFA biosynthesis inhibitor acetochlor (2-chloro-N-[ethoxymethyl]-N-[2-ethyl-6-methyl-phenyl]acetamide; ACE) in the unfertilized cotton ovules (Wang et al., 2015a). The transcriptome 
sequencing of 0 DPA fiber-attached ovule epidermal cells from Xu142, Xu142 $f l$, and three representative lines with different fiber phenotypes, viz. Lint-Fuzz (LF), Lint-Fuzzless (LM), and Less Lint-Fuzzless (LL), led to the identification of the fiber initiation-related genes (Hu et al., 2018). In this study, the GhMYB25-like and three other R2R3-MYB TFs (D06G1439, A13G0689, and D13G0798), were observed to be preferentially expressed in fiber initials at 0 DPA stage (Hu et al., 2018). In G. hirsutum, the expansin gene family members GhEXPA4o, GhEXPA1A, and GhEXPA8h exhibited preferential expression during the fiber initiation stage, which indicates their possible involvement in the fiber initiation process (Lv et al., 2020). In $G$. arboreum, a homeodomain-leucine zipper IV family gene, GaHDl, has been shown to regulate the fiber initiation process (Ding et al., 2020). Fine mapping and alternative splicing analysis showed that an $\mathrm{AG} \rightarrow \mathrm{AC}$ nucleotide substitution at a splicing site in $\mathrm{GaHD}$ l cause the alternative $3^{\prime}$ splicing error and leads to the fiberless seeds and glabrous stems phenotypes exhibited by the G. arboreum SMA-4 mutant, due to the dysfunctional GaHD1. The $\mathrm{H}_{2} \mathrm{O}_{2}$ production and $\mathrm{Ca}^{2+}$ flux analysis in wild type and SMA-4 mutant cotton plants indicated the potential involvement of GaHD1 in regulating the cellular redox and $\mathrm{Ca}^{2+}$ signaling during the fiber initiation process (Ding et al., 2020).

\section{Phytohormones play crucial roles in fiber cell initiation}

The plant hormones or phytohormones are grouped into different classes, viz. auxins, abscisic acid, brassinosteroids, cytokinins, gibberellins, ethylene, jasmonic acid, nitric oxide, salicylic acid, and strigolactones based on their chemical structures (Davies 2010; Zwanenburg et al., 2016). In plants, these phytohormones are present in significantly low concentrations, ranging from fmol $\mathrm{g}^{-1}$ to $\mathrm{pmol} \mathrm{g}^{-1}$ fresh weight (Šimura et al., 2018). Besides the other biological processes (Weyers and Paterson, 2001; Santner and Estelle, 2009; Kamiya 2010), 
phytohormones play a crucial role in cotton fiber initiation and development. The auxins, brassinosteroids, gibberellins, and jasmonic acid promote fiber initiation, whereas the abscisic acid and cytokinins inhibit the growth of fiber initials on the ovule epidermis (Figure 2).

Auxin, brassinosteroids, gibberellins, and jasmonic acid act as positive regulators of cotton fiber initiation

To understand the role of auxin and gibberellins on ovule and fiber growth, the thymidine analogue, 5-bromo-2-deoxyuridine (BUdR), was used (Dhindsa 1978). The BUdR arrest cell differentiation but allows cell growth to continue. The use of BUdR caused around a $70 \%$ reduction in fiber growth, but it allowed the ovule growth to continue. The use of an anti-auxin compound, p-chlorophenoxyisobutyric acid (PCIB), completely abolished the fiber initiation process, while the unfertilized ovules continued to grow in size (Dhindsa 1978). The application of AMO-1618 (2-isopropyl-4-dimethylamino- 5-methylphenyl- 1-piperidine carboxylate methyl chloride), a growth retardant, slightly reduced the fiber growth and dry weight when used with GA3, but it had a noticeable reduction in fiber growth and dry weight when used with IAA (Dhindsa 1978). The results indicated the role of gibberellin in ovule growth, and the involvement of IAA in fiber initiation and development (Dhindsa 1978). Gibberellin 20-oxidases (GA20ox) play an important role in the oxidation reactions during the gibberellin (GA) biosynthesis pathways (Plackett et al., 2012). Three G. hirsutum GA20ox homologous genes were identified and named GhGA20oxl to 3. Among these, the GhGA20ox 1 was preferentially expressed in the fibers (Xiao et al., 2010). The overexpression of GhGA20oxl resulted in more fiber initials in +2 and +3 DPA ovules of the transgenic cotton plants. The total amount of bioactive gibberellins (GAs), GA1 and GA4, was significantly increased in the GhGA20oxl overexpressing ovules and fiber tissues of the transgenic cotton plants. Light microscopy and 
scanning electron microscopy (SEM) analyses confirmed that GhGA20oxl overexpressing plants produced more fiber initials than the wild-type control plants. The results indicated that GhGA20oxl is involved in fiber cell initiation and elongation through the GA signaling (Xiao et al., 2010). The role of auxin in cotton fiber initiation was ascertained by its accumulation in the fiber initials on the anthesis. The anti-IAA antibody confirmed a higher accumulation of auxin in the fiber initials over the ovule epidermal layer on the day of anthesis (Zhang et al., 2011a). Further, the iaaM gene fused to a tissue-specific promoter from the petunia MADS-box gene, floral binding protein 7 (FBP7), led to an increase in the iaaM transcript levels and auxin content in the ovule epidermal cells of transgenic lines of cotton (Zhang et al., 2011a). Compared to the wild-type plants, the transgenic lines showed increased fiber initials and lint percentage. However, the $F B P 7$ promoter fused with iaaL (which produces biologically inactive auxin), the IAA content, and the number of fiber initials were significantly reduced in 0 DPA ovules (Zhang et al., 2011a). The $\beta$-glucuronidase (GUS) staining of -1 to +2 DPA transgenic cotton ovules, harboring the auxin-responsive reporter DR5::GUS, indicated the preferential accumulation of auxin in the ovule nucellus on -1 DPA (Zhang et al., 2017). The GUS staining was observed on the ovule surface at 0 DPA and also in the fiber cells of +1 and +2 DPA. The effect of auxin efflux and influx in cotton fiber initiation was assessed by treating the -1 DPA cotton ovules with auxin efflux inhibitor compounds like 1-N-naphthylphthalamic acid (NPA) and brefeldin A (BFA), and auxin influx inhibitor compound such as 1-naphthoxyacetic acid (NOA) (Zhang et al., 2017). After three days of culture, compared to the NOA-treated ovules, the fiber initials and the GUS staining was not observed in the NPA- and the BFA-treated ovules. The results indicated that instead of influx, the auxin efflux plays a critical role in fiber initiation (Zhang et al., 2017). The PIN-formed (PIN) proteins play a crucial role in auxin efflux (Petrášek et al., 
2006). The RNAi-mediated inhibition of GhPINIa suppressed fiber initiation and elongation in cotton ovules (Zhang et al., 2017). The GhPIN3a was found to be a key gene regulating auxin transport during the fiber cell initiation (Zeng et al., 2019). Its suppression confirmed the role of GhPIN3a in fiber initiation through the RNAi approach. The 3' UTR of GhPIN3a was placed downstream to an ovule epidermis-specific promoter proBAN. The expression of proBAN::GhPIN3a-RNAi construct led to severe inhibition of fiber initiation in ovule epidermis (Zeng et al., 2019). Moreover, the plasma membrane of non-fiber cells showed GhPIN3a, whereas the fiber cells lacked it. Thus, the epidermal cells which do not form fibers export auxins to fiber cells, which accumulate auxin due to the lack of GhPIN3a expression, indicating its key role in auxin signaling during the cotton fiber initiation (Zeng et al., 2019). Another phytohormone, brassinosteroid, also plays a significant role in cotton fiber development (Sun et al., 2004; Sun et al., 2005). Exogenous application of the brassinosteroid biosynthesis inhibitor, brassinazole2001 (Brz), inhibited the fiber initiation and elongation (Sun et al., 2005). However, the fiber initiation and elongation were restored upon the addition of $0.1 \mu \mathrm{M}$ brassinolide (BL) along with $10 \mu \mathrm{M} \mathrm{Brz}$. The expression levels of key genes of fiber expansion such as xyloglucantransferase/hydrolase $(X T H)$, expansin $(E X P)$, arabinogalactan protein $(A G P), \beta$ tubulin 1 (GhTUB1), and acyl carrier protein $(A C P)$ were found to be increased significantly in the three days cultured ovules treated with BL, whereas suppressed in ovules treated with Brz. This increased expression was indicative of more fiber initials on the ovule surface (Sun et al.,

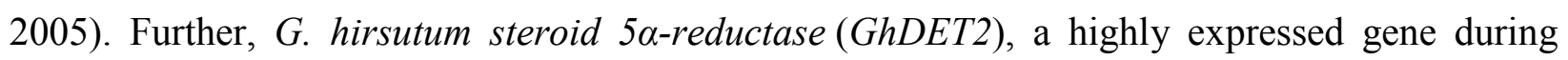
fiber initiation and elongation, has been implicated in the brassinosteroid-mediated regulation of fiber initiation and development (Luo et al., 2007). The silencing of GhDET2 using the antisense method led to the reduction in fiber cell initials and fiber cell elongation. The ectopic 
overexpression of GhDET2 driven by the promoter of a petunia-derived seed coatspecific FBP7 gene resulted in increased fiber initials on the ovule epidermis (Luo et al., 2007). The application of a biologically active brassinosteroid, epibrassinolide, led to the restoration of the effects caused by the suppression of GhDET2. The study thus indicated the role of brassinosteroid biosynthesis-related factors in cotton fiber initiation and development (Luo et al., 2007).

The members of the G. hirsutum allene-oxide cyclase (AOC) gene family, viz. GhAOC1, GhAOC2, GhAOC3, and GhAOC4, were observed to be expressed preferentially during the fiber initiation stage (Wang et al., 2015b). The AOC protein catalyzes an essential step during the jasmonic acid (JA) biosynthesis in plants (Schaller et al., 2008; Turner et al., 2002). The exogenous application of JA induced the expression of these AOCs, indicating their role in the JA-mediated regulation of cotton fiber initiation (Wang et al., 2015b). The JASMONATE ZIMDOMAIN (JAZ) proteins are the well-known repressors of the JA signaling in plants (Chini et al., 2007; Thines et al., 2007). In G. hirsutum, a JAZ family gene, GhJAZ2, was observed to be highly expressed during the fiber initiation stage. The GhJAZ2 overexpression inhibited the growth of fibers in the transgenic ovules. Thus, the GhJAZ2 overexpression negatively affected the fiber initiation, possibly through the jasmonate signaling (Hu et al., 2016). In G. hirsutum, a laccase gene, Ghlac1, has also been found to be involved in the JA-mediated regulation of cotton fiber initiation. The RNAi-mediated suppression of Ghlacl resulted in the hyperaccumulation of the JA and flavonoid contents in the transgenic ovules and fibers. The GhLacl RNAi lines exhibited increased fiber initials on the ovule epidermal cells. In the Ghlacl overexpression lines, due to the unaltered JA content, no effect was observed on the fiber initials. Therefore, the 
GhLacl-inhibition was speculated to promote fiber initiation through elevated JA accumulation (Hu et al., 2020).

\section{Abscisic acid and cytokinin negatively regulates the cotton fiber initiation}

The exogenous application of abscisic acid (ABA) has shown to inhibit the fiber cell initiation in the cultured ovules (Beasley and Ting, 1973). Compared to the wild-type ovules, the ABA significantly accumulated in 0 DPA ovules of short lint fiber producing Ligon lintless-2 (Li2) mutant (Gilbert et al., 2013). Thus, the abscisic acid was observed to regulate the fiber initiation negatively. The auxin and cytokinin were found to act antagonistically during the fiber initiation. The auxin levels were observed to be declined upon the rise in the cytokinin (CK) levels (Zeng et al., 2019). The enhanced CK levels severely affected the fiber initiation by suppressing the auxin accumulation. The treatment with a naturally occurring cytokinin, trans-zeatin (ZT), significantly suppressed the expression of auxin transporter GhPIN3a in 0 DPA ovules. The GUS signal reduced on the surface of the ovules of the proGhPIN3a::GUS transgenic plants upon treatment with $50 \mu \mathrm{M} \mathrm{ZT}$, and indicated the CK-mediated suppression of the GhPIN3a expression. The ZT treatment also disrupted the polar localization of the GhPIN3a::YFP in nonfiber cells of the ovule with the diminished GhPIN3a::YFP fluorescence intensity. The results indicated that excess CK antagonizes the auxin accumulation in the non-fiber cells through the repression of the GhPIN3a expression and delocalization (Zeng et al., 2019).

\section{The interplay of sugars significantly contributes in the cotton fiber initiation process}

Developing cotton fibers are metabolically highly active cells, thus, they work as an active nonphotosynthetic sink, which has a high demand for carbon in the form of sucrose for both as nutrients and energy (Ruan and Chourey, 1998; Ruan et al., 2003). Studies on fiber development in the last few decades suggested that import and utilization of sucrose in ovule epidermis play a 
crucial role during the early stages of fiber cell differentiation and development (Ruan and Chourey, 1998; Ruan et al., 2003; Wang et al., 2010; Wang et al., 2014a; Ahmad et al., 2019), as shown in Figure 2. Sugars can provide both signals and nutrients required for plant development (Ruan 2012). In plants, the sucrose synthase (Sus), a glycosyltransferase family enzyme, reversibly catalyzes the conversion of sucrose into UDP-glucose and fructose, whereas, the invertase (Inv), a hydrolase, irreversibly catalyzes the hydrolysis of sucrose into glucose and fructose (Stein and Granot, 2019). In plants, several isoforms of Sus and Inv are found in the cell wall and cytosol, but invertases are also specific to vacuoles (Stein and Granot 2019; Ruan 2012). Wang et al. (2010) identified specific vacuolar invertase (VIN1) in G. hirsutum, whose activity strongly increased at the early stage of fiber development (up to $70 \%$, at 0 and +1 DPA than -1 DPA). Comparative studies on fiberless mutant also demonstrated that sucrose transporters and sucrose synthase are highly expressed at the early stage of fiber development (Ruan and Chourey, 1998; Ruan et al., 2001; Ahmad et al., 2019). Furthermore, the suppression of the sucrose synthase (Sus) gene activity (Ruan et al., 2003) and GhVIN1 activity (Wang et al. 2014a) resulted in fiberless phenotypes. The ovules of the GhVIN1-RNAi lines produced fibers on their surface, if fructose and glucose were supplemented into the medium (Wang et al. 2014a). Sucrose synthase contributes to fiber commitment by supplying energy (ATP) via glycolysis (Ruan et al., 1997; Ruan et al., 2003). Additionally, fuzz fibers that initiate in the second wave of fiber initiation at approximately +3 DPA also require sucrose synthase (Ruan et al., 2005). Wang et al. (2014a) demonstrated that GhVIN1-mediated inhibition of fiber initiation is probably due to the lack of hexose signaling rather than the insufficiency of nutrients. They also reported that GhVIN1-mediated hexose signaling may be sensed by hexokinase, and it probably functions upstream to fiber initiation-related pathway genes. The 
rapid fiber elongation is driven by high turgor pressure achieved through the influx of water due to the high accumulation of glucose and fructose produced by sucrose synthase and invertase activity in the fiber cells (Ruan et al., 2001). Thus, these studies indicates the crucial roles of GhSus and GhVIN1 in early fiber cell commitment, differentiation, and growth.

\section{Transcription factors as key regulators of cotton fiber initiation}

In plants, the transcription factors (TFs) are well known to play a crucial role in diverse biological processes (Ramachandran et al., 1994; Yanagisawa 1998; Pandey and Somssich, 2009; Joshi et al., 2016). Several TFs expressed preferentially during the fiber initiation stages (Wang et al., 2004; Machado et al., 2009; Walford et al., 2011; Walford et al., 2012). The functional characterization of these TFs has indicated their potential involvement in the regulation of fiber initiation process (Table 1; Figure 2).

Table 1. List of cotton fiber initiation-related transcription factors.

\begin{tabular}{|c|c|c|}
\hline Transcription factor & Interacting partner & References \\
\hline GaMYB2 & RD22-like1 (RDL1) & Wang et al., 2004 \\
\hline $\begin{array}{l}\text { AT-Hook Transcription Factor } \\
\text { GhAT1 }\end{array}$ & FSltp4 & $\begin{array}{l}\text { Delaney et al., } \\
2007\end{array}$ \\
\hline GhMYB25 & - & $\begin{array}{l}\text { Machado et al., } \\
2009\end{array}$ \\
\hline $\begin{array}{c}\text { Gossypium barbadense Meristem } \\
\text { Layer } 1 \text { (GbML1) }\end{array}$ & $\begin{array}{l}\text { L1-box, GbML1 and } \\
\text { GbRDL1/GbMYB25 }\end{array}$ & Zhang et al., 2010 \\
\hline GhMYB25-like & - & $\begin{array}{l}\text { Walford et al., } \\
2011\end{array}$ \\
\hline GhHD1 & - & $\begin{array}{l}\text { Walford et al., } \\
2012\end{array}$ \\
\hline $\begin{array}{l}\text { PROTODERMAL FACTOR1 gene } \\
\text { (GbPDF1) }\end{array}$ & $\begin{array}{l}\text { PPIP1, (for putative GbPDF1 } \\
\text { interaction protein), PPIP2, and } \\
\text { PPIP3/HDZIP2ATATHB2 }\end{array}$ & Deng et al., 2012 \\
\hline
\end{tabular}


TEOSINTE-

BRANCHED1/CYCLOIDEA/PCF (TCP) GhTCP14

\author{
PIN2, IAA3, and AUX1 Wang et al., 2013
}

GhCPC

GhMYC1

Liu et al., 2015

JASMONATE ZIM-DOMAIN2 (JAZ2)

GhARF2 and GhARF18

\author{
GhMYB25-like, GhGL1, \\ GhMYC2, GhWD40 and GhJI1 \\ MYB2, RKY70, ERF106, and
}

Hu et al., 2016 bHLH144

The GaMYB2 TF, expressed predominantly during the early stages of cotton fiber development, was found to induce the production of Arabidopsis seed trichomes upon overexpression (Wang et al., 2004). GaMYB2 trans-activated the L1 box and MYB binding motif containing RD22like1 ( $R D L 1)$ gene promoter, and also showed to promote the trichome formation in the Arabidopsis trichome-less mutant gll (Wang et al., 2004). In another study, G. hirsutum AThook TF (GhAT1) found to interact with an AT-rich 84 bp fiber specificity region (FSR) present within the promoter region of a cotton lipid-transfer protein gene, FSltp4 (Delaney et al., 2007). The GhATl-mediated negative regulation of FSltp4 gene promoter in tobacco leaf trichomes suggested its role in cell fate determination during the fiber initiation (Delaney et al., 2007). The GhMYB25 expression found to reduce in the fiber-less mutant compared to the fiber-bearing wild-type cotton (Machado et al., 2009). GhMYB25 RNAi-silenced cotton plants yielded short fibers, reduced trichomes, and seed production, whereas the GhMYB25 overexpression promoted the fiber initiation and leaf trichome production. Thus, GhMYB25 facilitate the specialized outgrowths of cotton ovule epidermal cells during the cotton fiber initiation (Machado et al., 2009). G. barbadense Meristem Layer 1 (GbML1) interact with promoters of GbML1, GbRDL1 genes, and GbMYB25 TF (Zhang et al., 2010). Upon overexpression, GbML1 altered the epidermis and increased the leaf and stem trichomes in 
Arabidopsis. Therefore, GbML1 was proposed to control epidermal as well as fiber cell differentiation (Zhang et al., 2010). The GhMYB25-like, an R2R3 MYB, exhibited reduced expression in $X u 142 \mathrm{fl}$, a fiberless mutant of cotton (Walford et al., 2011). RNAi-mediated suppression of GhMYB25-like resulted in fiber-less seeds, whereas the extra copy had no obvious effect on fiber initials in the transgenic cotton. The GhMYB25-like thus observed to play a key role in the early stages of fiber initiation, but there may be some other factors that are likely to be involved along with GhMYB25-like in order to differentiate the ovule epidermal cells into the fiber cells (Walford et al., 2011). The cotton homeodomain leucine zipper (HD-ZIP) TF, GhHD1, was observed to be expressed preferentially in the early stages of fiber development (Walford et al., 2012). Upon GhHD-1 overexpression in cotton, an increment in fiber initials and fuzz percentage was observed, whereas, GhHD-1 silencing delayed the fiber initiation process. Along with the other cotton fiber development-related MYB factors, the GhHD-1 has been surmised to play a key role in cotton fiber initiation and progression by functioning downstream to the GhMYB25-like TF (Walford et al., 2012). G. barbadense PROTODERMAL FACTOR1 gene (GbPDF1), showed its highest expression in fiber cells at +5 dpa. The suppression of PDF1 resulted in shorter fibers and lower lint percentage in the transgenic lines (Deng et al., 2012). The PDF1 interact with PPIP1 (putative GbPDF1 interaction protein), PPIP2, and PPIP3. The ciselement HDZIP2ATATHB2 is essential for the $P D F 1$ expression. The higher accumulation of hydrogen peroxide $\left(\mathrm{H}_{2} \mathrm{O}_{2}\right)$ and the decreased expression of ethylene and pectin biosynthesisrelated genes in RNAi-suppressed transgenic lines indicated the critical role of GbPDF1 in fiber development via the $\mathrm{H}_{2} \mathrm{O}_{2}$ homeostasis, ethylene and pectin biosynthesis (Deng et al., 2012). The class I TEOSINTE-BRANCHEDI/CYCLOIDEA/PCF 14 (TCP14) TF expression increased upon supply of the exogenous auxin (Wang et al., 2013). It expressed preferentially in the initiation 
and elongating fiber cells, and promoted the initiation and elongation of trichomes and root hairs upon heterologous expression in Arabidopsis. GhTCP14 interact with the promoters of PINFORMED2 (PIN2), AUXIN/INDOLE-3-ACETIC ACID (IAA3), and AUXIN1 (AUX1) genes. Therefore, GbTCP14 is the potent regulator of auxin-mediated differentiation and elongation of the cotton fiber cells (Wang et al., 2013). GhCPC, an R3-MYB gene, was identified by the cDNA microarray analysis (Liu et al., 2015). It showed the differential expression between the TM-1 and the fuzzless-lintless mutants. GhCPC overexpression led to delayed fiber initiation and decreased fiber length. The yeast one-hybrid (Y1H) analysis showed the GhHOX3 interaction with GhMYC1. The GhCPC was suggested to negatively regulate the early fiber initiation and elongation via the CPC-MYC1-TTG1/4 complex (Liu et al., 2015). The cotton JASMONATE ZIM-DOMAIN (JAZ) protein-encoding gene GhJAZ2 overexpression inhibited the lint and the fuzz fiber initiation and reduced the cotton fiber length (Hu et al., 2016). GhJAZ2 negatively controls the lint and the fuzz fiber initiation in cotton by acting as a mediator of the JA signaling pathway by interacting with GhMYB25-like, GhGL1, GhMYC2, GhWD40, and GhJI1 (Hu et al., 2016). Two auxin response factors (ARFs) in G. hirsutum, GhARF2 and GhARF18, were observed to be the key regulators of auxin-mediated initiation of the cotton fibers (Xiao et al., 2018).

\section{Epigenetic regulation plays a pivotal role in cotton fiber initiation}

The epigenetic regulation generally leads to the heritable phenotypic changes in the gene expression without any modulation in the DNA sequence. The major epigenetic processes which regulate diverse biological processes in plants include DNA methylation (Zhang et al., 2006; Zilberman et al., 2007), histone modifications (Yu et al., 2011; Wang et al., 2014b), and small RNA-mediated modifications through the RNA-directed DNA methylation (RdDM) pathway 
(Wassenegger et al., 1994; Chan et al., 2004). In plants, the cytosine bases are generally methylated in $\mathrm{CG}, \mathrm{CHG}$, and $\mathrm{CHH}(\mathrm{H}=\mathrm{A}, \mathrm{C}$ or $\mathrm{T})$ sequence contexts (Cao and Jacobsen, 2002; Cokus et al., 2008; Lister et al., 2008). In Arabidopsis, the CG methylation is regulated by the METHYLTRANSFERASE 1 (MET1) (Ronemus et al., 1996; Finnegan et al., 1996), DECREASE IN DNA METHYLATION 1 (DDM1) (Gendrel et al., 2002; Brzeski and Jerzmanowski., 2003), and HISTONE DEACETYLASE 6 (HDA6) genes (Aufsatz et al., 2002; Murfett et al., 2001; Probst et al., 2004) whereas, the $\mathrm{CHG}$ and $\mathrm{CHH}$ methylation is regulated by CHROMOMETHYLASES3 (CMT3), DOMAINS REARRANGED METHYLTRANSFERASE1 (DRM1), and DOMAINS REARRANGED METHYLTRANSFERASE2 (DRM2) genes (Bartee et al., 2001; Cao and Jacobsen, 2002; Lindroth et al., 2001; Zilberman et al., 2004). DNA methylation and histone modification is shown to play an important role(s) in the cotton fiber initiation process (Figure 3). The role of DNA methylation in cotton fiber development was studied by treating the cotton ovule cultures with DNA (cytosine-5) methyltransferase inhibitor, zebularine (Wang et al., 2016). The inhibition of DNA methylation by zebularine led to the inhibition of fiber development. At 0 DPA, more than $95 \%$ of the cytosines were constitutively methylated in CG context followed by the $\mathrm{CHG}(89 \%)$ and the $\mathrm{CHH}(33 \%)$ context methylation. The CG and the CHG methylation patterns were constant in all-fiber developmental stages, while the CHH methylation significantly increased from the 0 DPA ovules to the 30 DPA fibers. This increase in $\mathrm{CHH}$ methylation leads to the conversion of the euchromatin into the heterochromatin, which leads to the transcriptional silencing of the fiber-related genes during the cotton fiber development. It was observed that the $\mathrm{CHH}$ methylation was higher in the Dt 


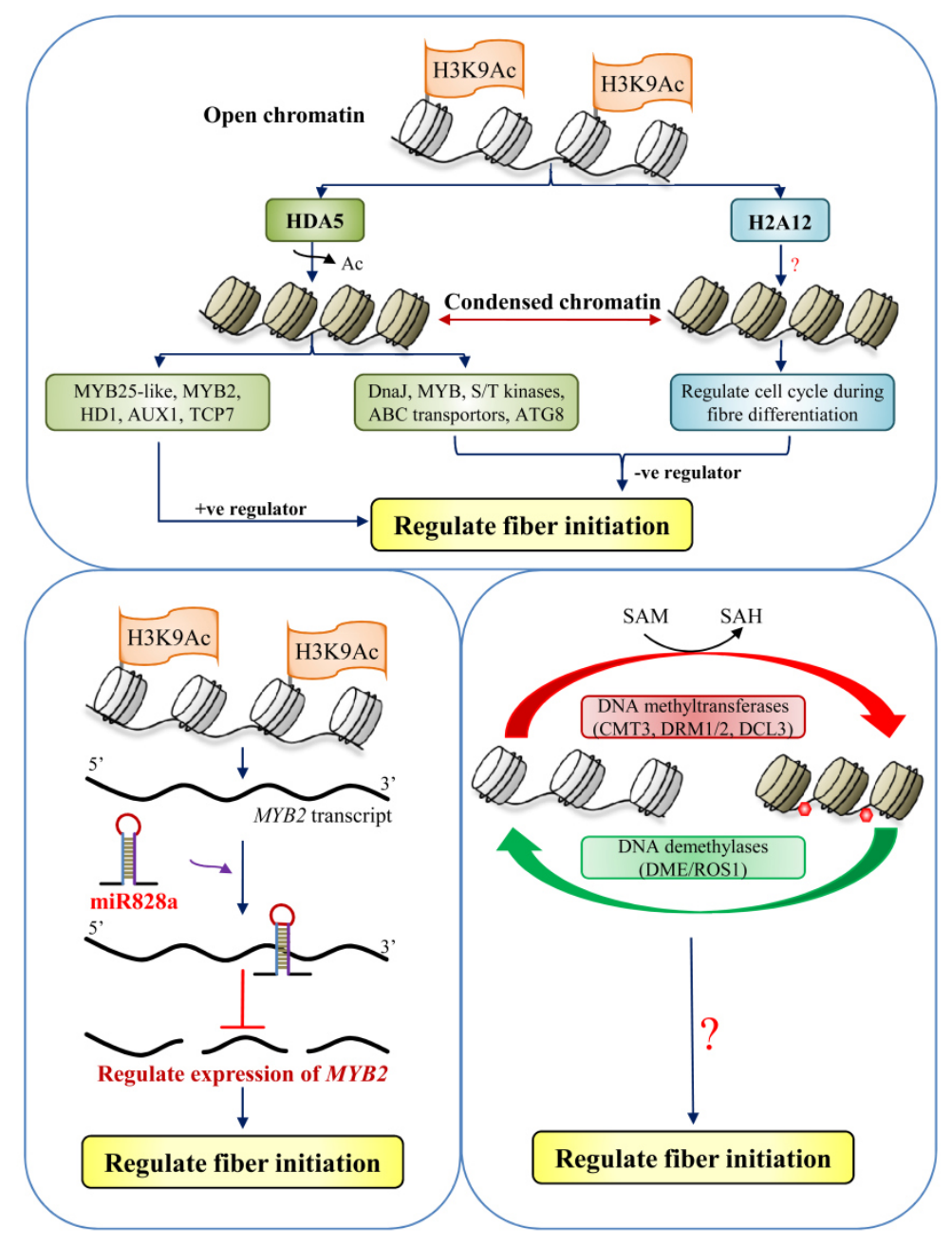

Figure 3. The epigenetic processes involved in the regulation of cotton fiber initiation. ATG8- Autophagy-related protein 8; AUX1- Auxin transporter protein 1; CMT3- DNA (cytosine-5)-methyltransferase CMT3; DCL3- Endoribonuclease dicer homolog 3; DMETranscriptional activator DEMETER; DRM1/2- Domains rearranged methylase 1/2; HD1- Zinc finger protein HD1; HDA5- Histone deacetylase 5; ROS1- Repressor of silencing 1; SAH- Sadenosylhomocysteine; SAM- S-adenosyl methionine; S/T kinases- Serine/threonine protein kinase; TCP7- Transcription factor TCP7.

subgenome, whereas, the $\mathrm{CG}$ and the $\mathrm{CHG}$ methylation was higher in the At subgenome (Wang et al., 2016). The RNA-directed DNA methylation (RdDM) pathway plays an essential role in the methylation of euchromatic regions ( $\mathrm{Du}$ et al., 2015), whereas the H3K9me2-dependent 
CMT2 pathway was found to mediate the $\mathrm{CHH}$ methylation in the heterochromatic regions (Zemach et al., 2013). The increase in $\mathrm{CHH}$ methylation led to the enrichment of the H3K9me2 modification marks and a sharp decrease in 24-nt siRNAs. This increase in CHH methylation indicates the role of $\mathrm{H} 3 \mathrm{~K} 9 \mathrm{me} 2$-dependent $\mathrm{CMT} 2$ pathway in the cotton fiber development (Wang et al., 2016). By using the methylation-sensitive amplified polymorphism (MSAP) and high-performance liquid chromatography (HPLC)-based assays, the DNA methylation diversity, genetic diversity, and changes in DNA methylation context were investigated in different cotton tissues, including the fibers at initiation stage (Osabe et al., 2014). Based on MSAP data, the CG, $\mathrm{CHG}$, and $\mathrm{CC}$ methylation patterns were analyzed to test the differences among the tissues. The CC methylation context exhibited significant differences. The 0 DPA ovule inner integument and 35 DPA fiber tissues showed the highest CC methylation, whereas, the 3 DPA ovules showed the lowest $\mathrm{CC}$ methylation. Compared to the $\mathrm{CC}$ methylation, the $\mathrm{CG}$ and the $\mathrm{CHG}$ methylation contexts found to be similar among different tissues. Among the eight G. hirsutum genotypes studied the total $\mathrm{CG}, \mathrm{CHG}$, and $\mathrm{CC}$ methylation found to be $49.7 \%$, whereas the average $\mathrm{CG}$, $\mathrm{CHG}$, and $\mathrm{CC}$ methylation was 37.8\%, 5.2\%, and 6.7\%, respectively (Osabe et al., 2014). Diversity analysis by simple matching (SM) coefficient showed that compared to the $G$. hirsutum genotypes, the $G$. barbadense genotypes were genetically more distant. The genetic diversity was low in comparison to the DNA methylation diversity in both the species. The CHG methylation context was more diverse than CG methylation in both the species (Osabe et. al., 2014).

By acting as histone modifiers, the histone acetyltransferases (HATs) and the histone deacetylases (HDACs) play an essential role in the transcriptional regulation (Strahal and Alis, 2000). Besides their regulatory roles in diverse plant-specific processes, the HDACs are involved 
in the regulation of the cotton fiber initiation (Figure 3). The role of a G. hirsutum histone deacetylase encoding gene, GhHDA5, has been established in the H3K9 deacetylation and fiber initiation (Kumar et al., 2018). GhHDA5 showed its preferential expression in -1 and 0 DPA cotton ovules. Cotton fiber initiation was inhibited upon treating the cultured cotton ovules with HDAC inhibitor, trichostatin A (TSA). Thus, the cotton fiber initiation was dependent on the HDAC activity. In vitro assay for the HDAC activity showed that GhHDA5 deacetylated the H3K9Ac mark. RNAi-mediated suppression of the GhHDA5 activity led to the reduction in fiber initials and lint yield in transgenic lines. In GhHDA5-suppressed RNAi-lines, perturbation in the reactive oxygen species (ROS) homeostasis and increase in autophagic cell death was observed. Chromatin immunoprecipitation (ChIP)-qPCR assay revealed the H3K9 hyper-acetylation in the promoter region of some differentially expressed genes in the GhHDA5-suppressed transgenic cotton lines (Kumar et al., 2018). The role of a $G$. hirsutum histone $H 2 A$ gene, GhH2A12, has been elucidated in cotton fiber initiation and development (Hao et al., 2014). The $G h H 2 A 12$ preferentially expresses in the fiber initials. Upon overexpression of $G h H 2 A 12$, the fiber initials and mature fiber length were reduced. It was also observed that overexpression of $G h H 2 A 12$ reduced the G2/M cell proportion and downregulated the expression of the cell cycle checkpoint regulatory genes, and thus negatively affected the mitotic cell division. The RNAi-mediated downregulation of GhH2A12 had not exhibited any significant changes (Hao et al., 2014).

\section{MicroRNAs: the small but potential regulators of cotton fiber initiation}

MicroRNAs (miRNAs) are $\sim 21$ nucleotides long ribonucleic acid molecules which act in a spatio-temporal manner and impart significant influence over the transcriptional and the posttranscriptional gene expression regulation in plants (Reinhart et al., 2002; Bartel, 2004; Jones- 
Rhoades et al., 2006; Meng et al., 2011; Meyers and Axtell, 2019). Several miRNAs have been identified, which are explicitly expressed during cotton fiber initiation and regulate the cotton fiber development by targeting the crucial genes associated with the cotton fiber initiation process.

\section{Identification of cotton fiber initiation-related miRNAs}

High-throughput or deep sequencing-based approaches have played a significant role in the identification of cotton fiber initiation and development-related miRNAs. To date, the majority of the fiber initiation-specific miRNAs have been identified by using these approaches. The first high-throughput or deep sequencing of small RNA libraries prepared from the wild-type and fuzz/lintless $(f l)$ mutant cotton ovule ( +1 to +10 DPA) tissues, led to the identification of 111 miRNA candidates that belong to 22 conserved miRNA families (Kwak et al., 2009). In cotton ovules $(-3,0,+3$ DPA) and leaf tissues, a total of 28 miRNAs, which include 27 conserved and 01 novel miRNA candidates, were identified by using the high-throughput sequencing approach by Pang et al. (2009). Deep sequencing of $-3,-1,0,+1$, and +3 DPA small RNA libraries led to the identification of 41 conserved miRNA families and 36 novel G. hirsutum miRNAs (Wang et al., 2012). A total of 28 known and five novel miRNAs were identified by deep sequencing of small RNA libraries prepared from $-3,-1,0,+1,+3$, and +5 DPA cotton ovules (Zhang et al., 2013). In G. barbadense, 47 conserved miRNA families and seven candidate miRNAs were identified in ovules $(-3,0,+3$ DPA) and fibers $(+7,+12,+20,+25$ DPA) (Liu et al., 2014). Small RNA sequencing of -1 to +1 DPA ovules and young leaves identified 65 conserved miRNA families in G. hirsutum (Xie et al., 2015). The deep sequencing of small RNA libraries prepared from $-3,0$, and +3 DPA ovules, treated with Indole-3-acetic acid (IAA) and without treatment (control), led to the identification of 58 known and 83 novel miRNAs (Zhao et al., 2019). 


\section{The predicted functional roles of miRNAs in cotton fiber initiation}

Several fiber initiation-specific miRNAs have been identified which exhibit their spatio-temporal expression from -3 to +3 DPA stages in cotton ovules (Pang et al., 2009; Wang et al., 2012; Zhang et al., 2013; Liu et al., 2014; Xie et al., 2015; Zhao et al., 2019). In most of the studies, the expression of miRNAs compared to their targets, or the differential expression of miRNAs upon treatment with phytohormones such as IAA, formed the basis for the identification of miRNAs specifically expressed during the different cotton fiber initiation $(-3$ to +3 DPA $)$ stages. The identified miRNAs targeted several genes that have been speculated to play potential roles during the cotton fiber initiation (Table 2).

Table 2. List of cotton fiber initiation-related miRNAs and their targets.

\begin{tabular}{|c|c|c|}
\hline $\operatorname{miRNA}$ & Target & References \\
\hline 156 & $\begin{array}{l}\text { Squamosa promoter-binding protein-like } 9 \\
\text { (SPL9) }\end{array}$ & $\begin{array}{l}\text { Wang et al., 2012; Zhang et al., } \\
2013 \text { Zhao et al., } 2019\end{array}$ \\
\hline $156 \mathrm{c}$ & Cytokinin response1 (CRE1) & Zhao et al., 2019 \\
\hline 160 & Auxin response factor 3 (ARF3) & $\begin{array}{l}\text { Wang et al., 2012; Zhang et al., } \\
2013\end{array}$ \\
\hline $160-3 p .2$ & GASA & Wang et al., 2012 \\
\hline 166 & $\begin{array}{l}\text { Class III HD-ZIP family of transcription } \\
\text { factors }\end{array}$ & $\begin{array}{l}\text { Pang et al., 2009; Wang et al., } \\
2012\end{array}$ \\
\hline 167 & LIM & $\begin{array}{l}\text { Wang et al., 2012; Zhang et al., } \\
2013\end{array}$ \\
\hline 171 & Scarecrow-like (SCL) & Wang et al., 2012 \\
\hline 390 & $\begin{array}{l}\text { Leucine-rich receptor-like protein kinase } \\
\qquad(\mathrm{RLK})\end{array}$ & Liu et al., 2014 \\
\hline 393 & Transport inhibitor responsel (TIR1) & Zhao et al., 2019 \\
\hline 397 & Laccase & Wang et al., 2012 \\
\hline
\end{tabular}




$\begin{array}{ccc}828 & \text { MYB2 } & \text { Guan et al., } 2014 \\ 858 & \text { MYB2 } & \text { Guan et al., } 2014 \\ 7504 \mathrm{a} & \text { pre-mRNA splicing factor (PSF) } & \text { Zhao et al., } 2019 \\ \text { nmiR3 } & \text { Cytochrome c oxidase subunit 1 (CO1) } & \text { Liu et al., } 2014 \\ 10 & \text { MYB33 } & \text { Zhao et al., } 2019 \\ \text { n22 } & \text { pre-mRNA splicing factor (PSF) } & \text { Wang et al., } 2012 \\ 36 & \text { Basic leucine zipper domain (bZIP) } & \text { Zhao et al., } 2019\end{array}$

A total of 07 miRNAs were found to be associated with fiber initiation (Wang et al., 2012). Among them, GhmiR156, GhmiR171, GhmiR160, GhmiR160-3p.2, and GhmiR167 showed less expression compared to their target mRNAs, whereas, GhmiR397 and GhmiRn22 expression was comparatively higher than their targets during the fiber initiation process (Wang et al., 2012). The target gene of GhmiR156, squamosa promoter-binding protein-like 9 (SPL9), was upregulated from -3 to +3 DPA. Since the SPL9 control the trichome formation in $A$. thaliana (Yu et al., 2010), it is speculated that SPL9 might be involved in the regulation of the fiber initiation process (Wang et al., 2012). GhmiR171 targets the scarecrow-like (SCL) gene. In A. thaliana, scarecrow-like 3 (SCL3) attenuates the DELLA proteins and promotes gibberellin (GA) biosynthesis gene expression (Zhang et al., 2011b). A GA-induced gene, GASA, was predicted to be targeted by GhmiR160-3p.2. Taken together, GhmiR171 and GhmiR160-3p.2 might be involved in the GA-mediated regulation of fiber initiation (Wang et al., 2012). Indole3-acetic acid (IAA) belongs to the auxin class of phytohormones that promote fiber initiation (Beasley and Ting, 1973). The auxin response factor 3 (ARF3) is targeted by GhmiR160, which might be involved in controlling the auxin signaling cascade during the fiber initiation (Wang et al., 2012). The GhmiR167 targeted a LIM-domain protein, acting as actin bundlers (Papuga et 
al., 2010). The increase in LIM protein expression from +1 to +3 DPA stages suggested its role in actin-assisted post-anthesis fiber initiation and development (Wang et al., 2012). Laccase, a target of GhmiR397, is mainly associated with lignin biosynthesis in plants (Ranocha et al., 2002). The reduced expression of laccase and thus, the less lignin content promote fiber bulging and initiation by the cotton ovule epidermal cell wall loosening (Wang et al., 2012). A novel miRNA, GhmiRn22, was observed to target the pre-mRNA splicing factor (PSF). The elevated expression of PSF in the $f l$ mutant in comparison to the wild-type cotton, suggests its negative role in fiber initiation (Wang et al., 2012). The class III HD-ZIP family of TFs, which play critical roles in organ polarity and morphogenesis (Rubio-Somoza and Weigel, 2011; Byrne 2006), were predicted to be targeted by miR166, which highly accumulated in the young cotton ovules and fibers (Zhang et al., 2013). The miR166 might be involved in regulating the ovule morphogenesis during the initiation and developmental stages. The miRNA172 was suggested to be another cotton ovule development-associated small RNA (Zhang et al., 2013), which functions together with miR156 (Wu et al., 2009). G. hirsutum MYB2, a target of miR828 and miR858, has been shown to promote Arabidopsis trichome formation (Guan et al., 2014). GhMYB2 role in Arabidopsis trichome initiation and development was similar to the previously reported G. arboreum MYB TF, GaMYB2 (Wang et al., 2004). Since the Arabidopsis trichome and cotton fiber initiation processes share some similarities, the miRNA828 has been speculated to play an important role in the cotton fiber initiation process by targeting GhMYB2 (Guan et al., 2014). In G. barbadense, Gb-miR390 showed higher expression during the fiber initiation stage and downregulated the expression of its target leucine-rich receptor-like protein kinase $(R L K)$ gene transcripts (Liu et al., 2014). Gb-miR390 has been speculated to promote fiber cell differentiation and initiation by downregulating its target gene (Liu et al., 
2014). Cytochrome c oxidase subunit 1 (CO1), a key oxidase in the respiratory chain, was targeted by nmiR3. The expression of $C O 1$ increased in the cotton ovules from -3 to +3 DPA, while the nmiR3 expression was decreased. The nmiR3 was predicted to affect fiber initiation by regulating the energy supply (Liu et al., 2014). The differentially expressed miRNAs and their targets in auxin (IAA)-treated and wild-type (control) were identified in $-3,0$, and +3 DPA ovules, which revealed potential miRNAs involved in the cotton fiber initiation (Zhao et al., 2019). Upon exogenous IAA application, the expression of ghr-miR10, ghr-miR36, ghr-miR156, ghr-miR156c, ghr-miR393, and ghr-miR7504a varied between the $-3,0$, and +3 DPA stages, in comparison to the untreated controls (Zhao et al., 2019). The expression of their target genes, viz. MYB33, basic leucine zipper domain (bZIP), SPL9, cytokinin response 1 (CRE1), transport inhibitor response 1 (TIR1), and PSF, respectively, were found to be contrasting (Zhao et al., 2019).

\section{Thrust areas that demand special attention to unravel the molecular processes governing the fiber initiation and fate determination}

The recent technological advancements have significantly facilitated the identification of several genetic and epigenetic factors controlling the initiation of fibers from the cotton ovule epidermal cells. The role of some phytohormones in cotton fiber initiation has also been reported. Despite the available information, there are still some important questions that need to be answered in order to precisely understand the molecular mechanisms which control the fiber initiation process. Being the cotton fiber cell fate-determining phase, the cotton fiber initiation is considered as one of the most crucial phase during the cotton fiber development. The most important aspect of cotton fiber initiation research is to unravel the biological processes involved in the commitment of ovule epidermal cells into fiber or non-fiber producing cells. Another 
important question arises about the genetic and epigenetic factors, which determine the fate of fiber producing cells, and the mechanisms by which they are operated to guide the nascent ovule epidermal cells to further develop into lint or fuzz fibers. Till now, no single factor is reported that could be solely responsible for the cell initiation and commitment in any aspects of fiber cell biology. Therefore, it's highly significant to comprehend the entire genetic, epigenetic, and phytohormone-mediated regulatory networks that determine the fiber commitment and initiation from the ovule epidermal cells. The sucrose synthase (SuSy) enzyme has been speculated to play an important role in fiber initiation by providing energy and precursors for the biosynthetic precursors through sucrose metabolism. Osmotically active solutes such as sugars, $\mathrm{K}^{+}$, and malate maintain the turgor potential in fiber cells (Basra and Malik, 1984). The cell turgor controlling genes have showed increased expression during the early stages of fiber initiation (Smart et al., 1998). Due to its high levels during the fiber initiation stage, the SuSy has been speculated to be involved in maintaining the high turgor potential by cleaving the sucrose into fructose and UDP-glucose, which significantly increases the osmotic contribution of sucrose, which might assist the protrusion of the fiber cell initials on the outer integument of ovule cells. The low levels of SuSy, and thus the low turgor, might be responsible for the lack of fiber initials on the fls mutant ovules (Ruan and Chourey, 1998). To completely understand the role of sugar metabolism in fiber initiation, besides SuSy, the other regulatory factors which contribute to the maintenance of cell turgor and osmotic regulation during the fiber initiation and fate determination needs to be investigated. Although the phytohormones have been reported to participate in fiber cell initiation, even so how these phytohormones involved in the commitment of ovule epidermal cells into fiber and non-fiber cells, is still not known. The elucidation of entire genetic and epigenetic factors and regulatory mechanisms involved in the plant hormone- 
mediated processes would help to precisely understand the role these phytohormones in fiber initiation and fate determination. There are several TFs such as MYB25 (Machado et al., 2009), MYB25-like (Walford et al., 2011), GhHD-1 (Walford et al., 2012), PDF1 (Deng et al., 2012), GhJAZ2 (Hu et al., 2016), etc., which are preferentially expressed during the fiber initiation stage and play an important role in the emergence of fiber initials from the cotton ovules. There are some other fiber initiation-related TFs, like GaMYB2 (Wang et al., 2004) and GhAT1 (Delaney et al., 2007), whose functions have been checked in the model plants such as Arabidopsis or tobacco. These TFs have been shown to affect the hair-like structure (trichome) formation in either leaves or seeds. To point out the clear depiction of the role of these TFs in fiber initiation, many explanatory works needs to be carried out in cotton. Although the overexpression or silencing of the TFs affects the fiber initiation, but do these TFs participate in fiber cell fate determination in either a direct or indirect way? If they, then how it works? The identification of the complete set of upstream or downstream targets of these initiation-related TFs might contribute to completely understand the fiber initiation and cell fate determination process. The chromatin immunoprecipitation-sequencing (ChIP-seq) can also be useful in providing information about the genome-wide targets of these initiation-specific TFs. How the small RNAs such as miRNAs precisely regulate these TFs during fiber initiation? What is their role in fiber cell fate determination? This query requires special attention, since till date no miRNA has been systematically characterized to investigate its role in cotton fiber initiation and ovule epidermal cell fate determination. How the epigenetic processes such as DNA methylation, histone modifications, and small RNA-mediated modifications participate in fiber cell initiation, is still a mystery. Much attention is required to unravel the role of epigenetic factors in cotton fiber initiation and cell fate determination. How the $\mathrm{CG}$ and $\mathrm{CHG}$ differentially methylated regions 
(DMRs) affect the fiber initiation and fate determination? Does the homoeolog expression bias play any role in fiber cell initiation and fate determination? How the $\mathrm{CG}, \mathrm{CC}, \mathrm{CHH}$, and $\mathrm{CHG}$ methylation pattern is related to the homoeolog expression bias during fiber initiation? Additionally, to get the answer for these unresolved questions, the identification and the elucidation of the roles of all the histones and histone modifiers, like the HATs and the HDACs, would help to better understand the cotton fiber initiation and fate determination process. The significant enrichment of some miRNAs during cotton fiber initiation stage indicates their crucial roles in fiber cell initiation and commitment. Several miRNAs have been identified which are significantly expressed during the cotton fiber initiation stage (Wang et al., 2012; Zhang et al., 2013; Xie et al., 2015). The potential targets of these identified miRNAs have also been predicted. However, their precise role in fiber initiation and commitment of ovule epidermal cells, into the fiber or non-fiber cells, is not understood.

\section{Conclusions and Future perspectives}

In conclusion, the complete information about the genetic and the epigenetic factors, as well as the phytohormones, would assist to precisely understand the initiation and fate determination of fiber cells. It would facilitate the precise manipulation of cotton fiber cell initiation and fate determining genetic and epigenetic factors through genetic or genome engineering tools to increase the number of the lint fiber producing committed cells on the ovule epidermal surface for the better fiber yield and quality traits. The fiber genomics has assisted to identifying the fiber initiation and development-related genes and other regulatory factors. The availability of high-quality reference genome would not only facilitate to understand the genomic basis of fiber initiation, but would also be helpful in the accurate identification and map-based positional cloning of the genes involved in determining the qualitative traits, such as the lint or the fuzz 
fiber initiation from the cotton ovule epidermal cells. The miRNAs were considered non-coding in nature until they were reported to code for the actively translated small peptides, known as the microRNA-encoded peptides (miPEPs) (Lauressergues et al., 2015). Do these miPEPs have any role in fiber initiation and cell fate determination? To get the answer to this question, it is necessary to identify and characterize the miPEPs associated with the fiber initiation and fate determination in cotton ovules. The cotton fiber being a single cell is an excellent model for singular cell studies. Single-cell transcriptomics is expected to provide the complete information of the expressed transcripts in the individual fiber producing (committed) and non-fiber producing (non-committed) cells during the initiation of cotton fiber cells from ovule epidermal cells. Single-cell microRNA-mRNA co-sequencing (Wang et al., 2019) is expected to assist in identifying the stage-specific miRNAs and their target transcripts in the fiber producing and the non-fiber producing cotton ovule cells. Besides overexpression and silencing, the CRISPR/Casmediated editing of the initiation and development-specific potential genes, TFs, miRNAs, and miPEPs is also a promising approach for the genomic modulation of the cotton species to achieve enhanced fiber initials, the higher percentage of lint fibers, and other quality parameters, like optimum micronaire value (3.7 to 4.2$)$ and high tensile strength.

\section{Outstanding questions}

- What are the biological events that determine the commitment of ovule epidermal cells to differentiate into fiber or non-fiber cells?

- How the fiber cells are genetically programmed to differentiate further into lint or fuzz fibers? 
- Although several genetic and epigenetic factors, as well as phytohormones, are known to participate in fiber initiation, however, how their mutual interactions orchestrate the fiber initiation, commitment, and fate determination?

- Besides the known genetic, epigenetic regulators, and phytohormones, what are the other regulatory factors which participate in fiber cell initiation and commitment?

- Although fiber-initiation related miRNAs and their targets have been reported, how they precisely regulate the fiber initiation? Do these fiber-initiation related miRNAs encode small peptides (miPEPs), which could participate in the fiber cell initiation and commitment?

- Could the single-cell studies help to unravel the complex molecular events and facilitate the genetic modulation of fiber initiation and fate determination?

\section{Author contributions}

SVS conceived the theme. All the authors contributed in the literature survey and data compilation to prepare the manuscript. The first author and SVS wrote the manuscript. The first author, RS, and VKT prepared the figures. SVS edited and finalized the manuscript. All the authors have checked and approved the finalized manuscript.

\section{Acknowledgements}

The authors acknowledge the Council of Scientific and Industrial Research, Govt. of India. The authors are grateful to the Director, CSIR-NBRI, for encouragement and support. The CSIRNBRI allotted manuscript number is "CSIR-NBRI_MS/2020/10/05".

\section{Conflict of interest statement}

The authors declare that they have no conflict of interest.

\section{References}

Ahmed M, Akhtar S, Fanglu M, Hasan MM, Shahid AA, Yanang X, Sarwar MB, Rao AQ, Husnain T, Wang X (2019) Sucrose Synthase (SuSy) Gene Expression: An Indicator for Cotton Fiber Initiation and Early Development. Russian Journal of Plant Physiology 66: 4149. 
Aufsatz W, Mette MF, van der Winden J, Matzke M, Matzke AJ (2002) HDA6, a putative histone deacetylase needed to enhance DNA methylation induced by double-stranded RNA. The EMBO Journal 21: 6832-6841.

Bartee L, Malagnac F, Bender J (2001) Arabidopsis cmt3 chromomethylase mutations block non-CG methylation and silencing of an endogenous gene. Genes \& Development 15: 17531758.

Bartel DP (2004) MicroRNAs: genomics, biogenesis, mechanism, and function. Cell 116: 28197.

Basra AS, Malik CP (1984) Development of the cotton fiber. International Review of Cytology 89: 65-113.

Beasley CA, Ting IP (1973) The effects of plant growth substances on in vitro fiber development from fertilized cotton ovules. American Journal of Botany 60: 130-139.

Berkley EE (1948) Cotton-A Versatile Textile Fiber. Textile Research Journal 18: 71-88.

Brzeski J, Jerzmanowski A (2003) Deficient in DNA methylation 1 (DDM1) defines a novel family of chromatin-remodeling factors. Journal of Biological Chemistry 278: 823-828.

Byrne ME (2006) Shoot meristem function and leaf polarity: the role of class III HD-ZIP genes. PLoS Genetics 2: e89.

Cao X, Jacobsen SE (2002) Locus-specific control of asymmetric and CpNpG methylation by the DRM and CMT3 methyltransferase genes. Proceedings of the National Academy of Sciences 99: 16491-16498.

Chan SW, Zilberman D, Xie Z, Johansen LK, Carrington JC, Jacobsen SE (2004) RNA silencing genes control de novo DNA methylation. Science 303: 1336-1336.

Chini A, Fonseca S, Fernandez G, Adie B, Chico JM, Lorenzo O, Garcia-Casado G, LópezVidriero I, Lozano FM, Ponce MR, Micol JL, Solano R (2007) The JAZ family of repressors is the missing link in jasmonate signalling. Nature 448: 666-671.

Cokus SJ, Feng S, Zhang X, Chen Z, Merriman B, Haudenschild CD, Pradhan S, Nelson SF, Pellegrini M, Jacobsen SE (2008) Shotgun bisulphite sequencing of the Arabidopsis genome reveals DNA methylation patterning. Nature 452: 215-219.

Davies PJ (2010) The plant hormones: their nature, occurrence, and functions. In: Plant Hormones (Davies PJ, eds), pp 1-15. Dordrecht: Springer.

Delaney SK, Orford SJ, Martin-Harris M, Timmis JN (2007) The fiber specificity of the cotton FSltp4 gene promoter is regulated by an AT-rich promoter region and the AT-hook transcription factor GhAT1. Plant and Cell Physiology 48: 1426-1437.

Deng F, Tu L, Tan J, Li Y, Nie Y, Zhang X (2012) GbPDF1 is involved in cotton fiber initiation via the core cis-element HDZIP2ATATHB2. Plant Physiology 158: 890-904.

Dhindsa RS (1978) Hormonal regulation of cotton ovule and fiber growth: Effects of bromodeoxyuridine, AMO-1618 and p-chlorophenoxyisobutyric acid. Planta 141: 269-272. 
Ding M, Cao Y, He S, Sun J, Dai H, Zhang H, Sun C, Jiang Y, Paterson AH, Rong J (2020) GaHD1, a candidate gene for the Gossypium arboreum SMA-4 mutant, promotes trichome and fiber initiation by cellular $\mathrm{H}_{2} \mathrm{O}_{2}$ and $\mathrm{Ca}^{2+}$ signals. Plant Molecular Biology 103: 409-423.

Du J, Johnson LM, Jacobsen SE, Patel DJ (2015) DNA methylation pathways and their crosstalk with histone methylation. Nature Reviews Molecular Cell Biology 16: 519-532.

Finnegan EJ, Peacock WJ, Dennis ES (1996) Reduced DNA methylation in Arabidopsis thaliana results in abnormal plant development. Proceedings of the National Academy of Sciences 93: 8449-8454.

Gendrel AV, Lippman Z, Yordan C, Colot V, Martienssen RA (2002) Dependence of heterochromatic histone $\mathrm{H} 3$ methylation patterns on the Arabidopsis gene DDM1. Science 297: 1871-1873.

Gilbert MK, Bland JM, Shockey JM, Cao H, Hinchliffe DJ, Fang DD, Naoumkina M (2013) A transcript profiling approach reveals an abscisic acid-specific glycosyltransferase (UGT73C14) induced in developing fiber of Ligon lintless-2 mutant of cotton (Gossypium hirsutum L.). PLoS One 8: e75268.

Graves DA, Stewart JM (1988) Chronology of the differentiation of cotton (Gossypium hirsutum L.) fiber cells. Planta 175: 254-258.

Guan X, Pang M, Nah G, Shi X, Ye W, Stelly DM, Chen ZJ (2014) miR828 and miR858 regulate homoeologous $M Y B 2$ gene functions in Arabidopsis trichome and cotton fibre development. Nature Communications 5: 3050.

Hao J, Chen S, Tu L, Hu H, Zhang X (2014) GhH2A12, a replication-dependent histone H2A gene from Gossypium hirsutum, is negatively involved in the development of cotton fiber cells. Plant Cell Reports 33: 1711-1721.

Hu H, He X, Tu L, Zhu L, Zhu S, Ge Z, Zhang X (2016) GhJAZ2 negatively regulates cotton fiber initiation by interacting with the R2R3-MYB transcription factor GhMYB25-like. The Plant Journal 88: 921-935.

Hu H, Wang M, Ding Y, Zhu S, Zhao G, Tu L, Zhang X (2018) Transcriptomic repertoires depict the initiation of lint and fuzz fibres in cotton (Gossypium hirsutum L.). Plant Biotechnology Journal 16: 1002-12.

Hu Q, Xiao S, Guan Q, Tu L, Sheng F, Du X, Zhang X (2020) The laccase gene GhLac1 modulates fiber initiation and elongation by coordinating jasmonic acid and flavonoid metabolism. The Crop Journal 8: 522-533.

Jones-Rhoades MW, Bartel DP, Bartel B (2006) MicroRNAs and their regulatory roles in plants. Annual Review of Plant Biology 57: 19-53.

Joshi R, Wani SH, Singh B, Bohra A, Dar ZA, Lone AA, Pareek A, Singla-Pareek SL (2016) Transcription factors and plants response to drought stress: current understanding and future directions. Frontiers in Plant Science 7: 1029.

Kamiya Y (2010) Plant Hormones: Versatile Regulators of Plant Growth and Development. Annual Review of Plant Biology 61. 
Kim HJ, Triplett BA (2001) Cotton fiber growth in planta and in vitro. Models for plant cell elongation and cell wall biogenesis. Plant Physiology 127: 1361-1366.

Kumar V, Singh B, Singh SK, Rai KM, Singh SP, Sable A, Pant P, Saxena G, Sawant SV (2018) Role of GhHDA5 in H3K9 deacetylation and fiber initiation in Gossypium hirsutum. The Plant Journal 95: 1069-1083.

Kwak PB, Wang QQ, Chen XS, Qiu CX, Yang ZM (2009) Enrichment of a set of microRNAs during the cotton fiber development. BMC Genomics 10: 457.

Lauressergues D, Couzigou JM, San Clemente H, Martinez Y, Dunand C, Bécard G, Combier JP (2015) Primary transcripts of microRNAs encode regulatory peptides. Nature 520: 90-93.

Lee JJ, Hassan OS, Gao W, Wei NE, Kohel RJ, Chen XY, Payton P, Sze SH, Stelly DM, Chen ZJ (2006) Developmental and gene expression analyses of a cotton naked seed mutant. Planta 223: 418-432.

Lindroth AM, Cao X, Jackson JP, Zilberman D, McCallum CM, Henikoff S, Jacobsen SE (2001) Requirement of CHROMOMETHYLASE3 for maintenance of CpXpG methylation. Science 292: 2077-2080.

Lister R, O'Malley RC, Tonti-Filippini J, Gregory BD, Berry CC, Millar AH, Ecker JR (2008) Highly integrated single-base resolution maps of the epigenome in Arabidopsis. Cell 133: 523-536.

Liu B, Zhu Y, Zhang T (2015) The R3-MYB gene GhCPC negatively regulates cotton fiber elongation. PLoS One 10: e0116272.

Liu N, Tu L, Tang W, Gao W, Lindsey K, Zhang X (2014) Small RNA and degradome profiling reveals a role for miRNAs and their targets in the developing fibers of Gossypium barbadense. The Plant Journal 80: 331-344.

Luo M, Xiao Y, Li X, Lu X, Deng W, Li D, Hou L, Hu M, Li Y, Pei Y (2007) GhDET2, a steroid $5 \alpha$-reductase, plays an important role in cotton fiber cell initiation and elongation. The Plant Journal 51: 419-430.

Lv LM, Zuo DY, Wang XF, Cheng HL, Zhang YP, Wang QL, Song GL, Ma ZY (2020) Genome-wide identification of the expansin gene family reveals that expansin genes are involved in fibre cell growth in cotton. BMC Plant Biology 20: 1-3.

Machado A, Wu Y, Yang Y, Llewellyn DJ, Dennis ES (2009) The MYB transcription factor GhMYB25 regulates early fibre and trichome development. The Plant Journal 59: 52-62.

Meng Y, Shao C, Wang H, Chen M (2011) The regulatory activities of plant microRNAs: a more dynamic perspective. Plant Physiology 157: 1583-1595.

Meyers BC, Axtell MJ (2019) MicroRNAs in Plants: Key Findings from the Early Years. The Plant Cell 31: 1206-1207.

Murfett J, Wang XJ, Hagen G, Guilfoyle TJ (2001) Identification of Arabidopsis histone deacetylase HDA6 mutants that affect transgene expression. The Plant Cell 13: 1047-1061.

Nigam D, Kavita P, Tripathi RK, Ranjan A, Goel R, Asif M, Shukla A, Singh G, Rana D, Sawant SV (2014) Transcriptome dynamics during fibre development in contrasting genotypes of Gossypium hirsutum L. Plant Biotechnology Journal 12: 204-218. 
Osabe K, Clement JD, Bedon F, Pettolino FA, Ziolkowski L, Llewellyn DJ, Finnegan EJ, Wilson IW (2014) Genetic and DNA methylation changes in cotton (Gossypium) genotypes and tissues. PLoS One 9: e86049.

Pandey SP, Somssich IE (2009) The role of WRKY transcription factors in plant immunity. Plant Physiology 150: 1648-1655.

Pang M, Woodward AW, Agarwal V, Guan X, Ha M, Ramachandran V, Chen X, Triplett BA, Stelly DM, Chen ZJ (2009) Genome-wide analysis reveals rapid and dynamic changes in miRNA and siRNA sequence and expression during ovule and fiber development in allotetraploid cotton (Gossypium hirsutum L.). Genome Biology 10: R122.

Papuga J, Hoffmann C, Dieterle M, Moes D, Moreau F, Tholl S, Steinmetz A, Thomas C (2010) Arabidopsis LIM proteins: a family of actin bundlers with distinct expression patterns and modes of regulation. The Plant Cell 22: 3034-3052.

Petrášek J, Mravec J, Bouchard R, Blakeslee JJ, Abas M, Seifertová D, Wiśniewska J, Tadele Z, Kubeš M, Čovanová M, Dhonukshe P (2006) PIN proteins perform a rate-limiting function in cellular auxin efflux. Science 312: 914-918.

Plackett AR, Powers SJ, Fernandez-Garcia N, Urbanova T, Takebayashi Y, Seo M, Jikumaru Y, Benlloch R, Nilsson O, Ruiz-Rivero O, Phillips AL (2012) Analysis of the developmental roles of the Arabidopsis gibberellin 20-oxidases demonstrates that GA20ox1,-2, and-3 are the dominant paralogs. The Plant Cell 24: 941-960.

Probst AV, Fagard M, Proux F, Mourrain P, Boutet S, Earley K, Lawrence RJ, Pikaard CS, Murfett J, Furner I, Vaucheret H (2004) Arabidopsis histone deacetylase HDA6 is required for maintenance of transcriptional gene silencing and determines nuclear organization of rDNA repeats. The Plant Cell 16: 1021-1034.

Ramachandran S, Hiratsuka K, Chua NH (1994) Transcription factors in plant growth and development. Current Opinion in Genetics \& Development 4: 642-646.

Ranocha P, Chabannes M, Chamayou S, Danoun S, Jauneau A, Boudet AM, Goffner D (2002) Laccase down-regulation causes alterations in phenolic metabolism and cell wall structure in poplar. Plant Physiology 129: 145-155.

Reinhart BJ, Weinstein EG, Rhoades MW, Bartel B, Bartel DP (2002) MicroRNAs in plants. Genes \& Development 16: 1616-1626.

Ronemus MJ, Galbiati M, Ticknor C, Chen J, Dellaporta SL (1996) Demethylation-induced developmental pleiotropy in Arabidopsis. Science 273: 654-657.

Ruan YL (2012) Signaling roles of sucrose metabolism in plant development. Molecular Plant 5: $763-765$.

Ruan YL, Chourey PS (1998) A fiberless seed mutation in cotton is associated with lack of fiber cell initiation in ovule epidermis and alterations in sucrose synthase expression and carbon partitioning in developing seeds. Plant Physiology 118: 399-406.

Ruan YL, Chourey PS, Delmer DP, Perez-Grau L (1997) The differential expression of sucrose synthase in relation to diverse patterns of carbon partitioning in developing cotton seed. Plant Physiology 115: 375-385. 
Ruan YL, Llewellyn DJ, Furbank RT (2001) The control of single-celled cotton fiber elongation by developmentally reversible gating of plasmodesmata and coordinated expression of sucrose and $\mathrm{K}^{+}$transporters and expansin. The Plant Cell 13: 47-60.

Ruan YL, Llewellyn DJ, Furbank RT (2003) Suppression of sucrose synthase gene expression represses cotton fiber cell initiation, elongation, and seed development. The Plant Cell 15: 952-964.

Ruan YL, Llewellyn DJ, Furbank RT, Chourey PS (2005) The delayed initiation and slow elongation of fuzz-like short fibre cells in relation to altered patterns of sucrose synthase expression and plasmodesmata gating in a lintless mutant of cotton. Journal of Experimental Botany 56: 977-984.

Rubio-Somoza I, Weigel D (2011) MicroRNA networks and developmental plasticity in plants. Trends in Plant Science 16: 258-264.

Santner A, Estelle M (2009) Recent advances and emerging trends in plant hormone signalling. Nature 459: 1071-1078.

Schaller F, Zerbe P, Reinbothe S, Reinbothe C, Hofmann E, Pollmann S (2008) The allene oxide cyclase family of Arabidopsis thaliana-localization and cyclization. The FEBS Journal 275: 2428-2441.

Shi YH, Zhu SW, Mao XZ, Feng JX, Qin YM, Zhang L, Cheng J, Wei LP, Wang ZY, Zhu YX (2006) Transcriptome profiling, molecular biological, and physiological studies reveal a major role for ethylene in cotton fiber cell elongation. The Plant Cell 18: 651-664.

Šimura J, Antoniadi I, Široká J, Tarkowská D, Strnad M, Ljung K, Novák O (2018) Plant hormonomics: multiple phytohormone profiling by targeted metabolomics. Plant Physiology 177: 476-489.

Smart LB, Vojdani F, Maeshima M, Wilkins TA (1998) Genes involved in osmoregulation during turgor-driven cell expansion of developing cotton fibers are differentially regulated. Plant Physiology 116: 1539-1549.

Stein O, Granot D (2019) An overview of sucrose synthases in plants. Frontiers in Plant Science 10: 95.

Stewart JM (1975) Fiber initiation on the cotton ovule (Gossypium hirsutum). American Journal of Botany 62: 723-730.

Strahl BD, Allis CD (2000) The language of covalent histone modifications. Nature 403: 41-45.

Sun Y, Fokar M, Asami T, Yoshida S, Allen RD (2004) Characterization of the brassinosteroid insensitive 1 genes of cotton. Plant Molecular Biology 54: 221-232.

Sun Y, Veerabomma S, Abdel-Mageed HA, Fokar M, Asami T, Yoshida S, Allen RD (2005) Brassinosteroid regulates fiber development on cultured cotton ovules. Plant and Cell Physiology 46: 1384-1391.

Taliercio EW, Boykin D (2007) Analysis of gene expression in cotton fiber initials. BMC Plant Biology 7: 22. 
Thines B, Katsir L, Melotto M, Niu Y, Mandaokar A, Liu G, Nomura K, He SY, Howe GA, Browse J (2007) JAZ repressor proteins are targets of the SCF COI1 complex during jasmonate signalling. Nature 448: 661-665.

Tu LL, Zhang XL, Liang SG, Liu DQ, Zhu LF, Zeng FC, Nie YC, Guo XP, Deng FL, Tan JF, $\mathrm{Xu}$ L (2007) Genes expression analyses of sea-island cotton (Gossypium barbadense L.) during fiber development. Plant Cell Reports 26: 1309-1320.

Turner JG, Ellis C, Devoto A (2002) The jasmonate signal pathway. The Plant Cell 14: S153164.

Walford SA, Wu Y, Llewellyn DJ, Dennis ES (2011) GhMYB25-like: a key factor in early cotton fibre development. The Plant Journal 65: 785-797.

Walford SA, Wu Y, Llewellyn DJ, Dennis ES (2012) Epidermal cell differentiation in cotton mediated by the homeodomain leucine zipper gene, GhHD-1. The Plant Journal 71: 464-478.

Wang L, Cook A, Patrick JW, Chen XY, Ruan YL (2014a) Silencing the vacuolar invertase gene GhVIN1 blocks cotton fiber initiation from the ovule epidermis, probably by suppressing a cohort of regulatory genes via sugar signaling. The Plant Journal 78: 686-696.

Wang L, Li XR, Lian H, Ni DA, He YK, Chen XY, Ruan YL (2010). Evidence that high activity of vacuolar invertase is required for cotton fiber and Arabidopsis root elongation through osmotic dependent and independent pathways, respectively. Plant Physiology 154: 744-756.

Wang L, Zhu Y, Hu W, Zhang X, Cai C, Guo W (2015b) Comparative transcriptomics reveals jasmonic acid-associated metabolism related to cotton fiber initiation. PLoS One 10:e0129854.

Wang M, Wang P, Tu L, Zhu S, Zhang L, Li Z, Zhang Q, Yuan D, Zhang X (2016) Multi-omics maps of cotton fibre reveal epigenetic basis for staged single-cell differentiation. Nucleic Acids Research 44: 4067-4079.

Wang MY, Zhao PM, Cheng HQ, Han LB, Wu XM, Gao P, Wang HY, Yang CL, Zhong NQ, Zuo JR, Xia GX (2013) The cotton transcription factor TCP14 functions in auxin-mediated epidermal cell differentiation and elongation. Plant Physiology 162: 1669-1680.

Wang N, Zheng J, Chen Z, Liu Y, Dura B, Kwak M, Xavier-Ferrucio J, Lu YC, Zhang M, Roden C, Cheng J (2019) Single-cell microRNA-mRNA co-sequencing reveals non-genetic heterogeneity and mechanisms of microRNA regulation. Nature Communications 10: 95.

Wang S, Wang JW, Yu N, Li CH, Luo B, Gou JY, Wang LJ, Chen XY (2004) Control of plant trichome development by a cotton fiber $M Y B$ gene. The Plant Cell 16: 2323-2334.

Wang XC, Li Q, Jin X, Xiao GH, Liu GJ, Liu NJ, Qin YM (2015a) Quantitative proteomics and transcriptomics reveal key metabolic processes associated with cotton fiber initiation. Journal of Proteomics 114: 16-27.

Wang Z, Cao H, Chen F, Liu Y (2014b) The roles of histone acetylation in seed performance and plant development. Plant Physiology and Biochemistry 84: 125-133.

Wang ZM, Xue W, Dong CJ, Jin LG, Bian SM, Wang C, Wu XY, Liu JY (2012) A comparative miRNAome analysis reveals seven fiber initiation-related and 36 novel miRNAs in developing cotton ovules. Molecular Plant 5: 889-900. 
Wassenegger M, Heimes S, Riedel L, Sänger HL (1994) RNA-directed de novo methylation of genomic sequences in plants. Cell 76: 567-576.

Weyers JD, Paterson NW (2001) Plant hormones and the control of physiological processes. New Phytologist 152: 375-407.

Wu G, Park MY, Conway SR, Wang JW, Weigel D, Poethig RS (2009) The sequential action of miR156 and miR172 regulates developmental timing in Arabidopsis. Cell 138: 750-759.

Wu Y, Machado AC, White RG, Llewellyn DJ, Dennis ES (2006) Expression profiling identifies genes expressed early during lint fibre initiation in cotton. Plant and Cell Physiology 47: $107-127$.

Xiao G, He P, Zhao P, Liu H, Zhang L, Pang C, Yu J (2018) Genome-wide identification of the GhARFgene family reveals that GhARF2 and GhARF18 are involved in cotton fibre cell initiation. Journal of Experimental Botany 69: 4323-4337.

Xiao YH, Li DM, Yin MH, Li XB, Zhang M, Wang YJ, Dong J, Zhao J, Luo M, Luo XY, Hou L (2010) Gibberellin 20-oxidase promotes initiation and elongation of cotton fibers by regulating gibberellin synthesis. Journal of Plant Physiology 167: 829-837.

Xie F, Jones DC, Wang Q, Sun R, Zhang B (2015) Small RNA sequencing identifies miRNA roles in ovule and fibre development. Plant Biotechnology Journal 13: 355-369.

Yanagisawa S (1998) Transcription factors in plants: physiological functions and regulation of expression. Journal of Plant Research 111: 363-371.

Yu CW, Liu X, Luo M, Chen C, Lin X, Tian G, Lu Q, Cui Y, Wu K (2011) HISTONE DEACETYLASE6 interacts with FLOWERING LOCUS $D$ and regulates flowering in Arabidopsis. Plant Physiology 156: 173-184.

Yu N, Cai WJ, Wang S, Shan CM, Wang LJ, Chen XY (2010) Temporal control of trichome distribution by microRNA156-targeted SPL genes in Arabidopsis thaliana. The Plant Cell 22: 2322-2335.

Zemach A, Kim MY, Hsieh PH, Coleman-Derr D, Eshed-Williams L, Thao K, Harmer SL, Zilberman D (2013) The Arabidopsis nucleosome remodeler DDM1 allows DNA methyltransferases to access H1-containing heterochromatin. Cell 153: 193-205.

Zeng J, Zhang M, Hou L, Bai W, Yan X, Hou N, Wang H, Huang J, Zhao J, Pei Y (2019) Cytokinin inhibits cotton fiber initiation by disrupting PIN3a-mediated asymmetric accumulation of auxin in the ovule epidermis. Journal of Experimental Botany 70: 31393151.

Zhang F, Zuo K, Zhang J, Liu X, Zhang L, Sun X, Tang K (2010) An L1 box binding protein, GbML1, interacts with GbMYB25 to control cotton fibre development. Journal of Experimental Botany 61: 3599-3613.

Zhang H, Wan Q, Ye W, Lv Y, Wu H, Zhang T (2013) Genome-wide analysis of small RNA and novel microRNA discovery during fiber and seed initial development in Gossypium hirsutum L. PLoS One 8: e69743.

Zhang M, Zeng JY, Long H, Xiao YH, Yan XY, Pei Y (2017) Auxin regulates cotton fiber initiation via GhPIN-mediated auxin transport. Plant and Cell Physiology 58: 385-397. 
Zhang M, Zheng X, Song S, Zeng Q, Hou L, Li D, Zhao J, Wei Y, Li X, Luo M, Xiao Y (2011a) Spatiotemporal manipulation of auxin biosynthesis in cotton ovule epidermal cells enhances fiber yield and quality. Nature Biotechnology 29: 453-458.

Zhang X, Yazaki J, Sundaresan A, Cokus S, Chan SW, Chen H, Henderson IR, Shinn P, Pellegrini M, Jacobsen SE, Ecker JR (2006) Genome-wide high-resolution mapping and functional analysis of DNA methylation in Arabidopsis. Cell 126:1189-1201.

Zhang ZL, Ogawa M, Fleet CM, Zentella R, Hu J, Heo JO, Lim J, Kamiya Y, Yamaguchi S, Sun TP (2011b) Scarecrow-like 3 promotes gibberellin signaling by antagonizing master growth repressor DELLA in Arabidopsis. Proceedings of the National Academy of Sciences 108: 2160-2165.

Zhao T, Xu X, Wang M, Li C, Li C, Zhao R, Zhu S, He Q, Chen J (2019) Identification and profiling of upland cotton microRNAs at fiber initiation stage under exogenous IAA application. BMC Genomics 20: 421.

Zilberman D, Cao X, Johansen LK, Xie Z, Carrington JC, Jacobsen SE (2004) Role of Arabidopsis ARGONAUTE4 in RNA-directed DNA methylation triggered by inverted repeats. Current Biology 14: 1214-1220.

Zilberman D, Gehring M, Tran RK, Ballinger T, Henikoff S (2007) Genome-wide analysis of Arabidopsis thaliana DNA methylation uncovers an interdependence between methylation and transcription. Nature Genetics 39: 61-69.

Zwanenburg B, Pospíšil T, Zeljković SĆ (2016) Strigolactones: new plant hormones in action. Planta 243: 1311-1326. 